\title{
Development of the Decoupled Discreet-Time Jacobian Eigenvalue Approximation for Situational Awareness Utilizing Open PDC
}

\author{
Sean D. Kantra, Elham B. Makram \\ Holcombe Department of Electrical and Computer Engineering, Clemson University, Clemson, USA \\ Email: skantra@g.clemson.edu,makram@clemson.edu
}

How to cite this paper: Kantra, S.D. and Makram, E.B. (2016) Development of the Decoupled Discreet-Time Jacobian Eigenvalue Approximation for Situational Awareness Utilizing Open PDC. Journal of Power and Energy Engineering, 4, 21-35. http://dx.doi.org/10.4236/jpee.2016.49003

Received: August 17, 2016

Accepted: September 5, 2016

Published: September 8, 2016

Copyright $\odot 2016$ by authors and Scientific Research Publishing Inc. This work is licensed under the Creative Commons Attribution International License (CC BY 4.0). http://creativecommons.org/licenses/by/4.0/

\begin{abstract}
With the increased number of PMUs in the power grid, effective high speed, realtime methods to ascertain relevant data for situational awareness are needed. Several techniques have used data from PMUs in conjunction with state estimation to assess system stability and event detection. However, these techniques require system topology and a large computational time. This paper presents a novel approach that uses real-time PMU data streams without the need of system connectivity or additional state estimation. The new development is based on the approximation of the eigenvalues related to the decoupled discreet-time power flow Jacobian matrix using direct openPDC data in real-time. Results are compared with other methods, such as Prony's method, which can be too slow to handle big data. The newly developed Discreet-Time Jacobian Eigenvalue Approximation (DDJEA) method not only proves its accuracy, but also shows its effectiveness with minimal computational time: an essential element when considering situational awareness.
\end{abstract}

\section{Keywords}

Synchrophasor, PMU, Open PDC, Power Flow Jacobian, Decoupled Discreet-Time Jacobian Approximation, Singular Value Decomposition (SVD), Prony Analysis

\section{Introduction}

The traditional power flow Jacobian matrix, utilized in Newton-Raphson method, cannot be calculated given these constraints. Analyzing the load flow Jacobian matrix for singular values has been previously utilized to show system weak areas and assess stability [1]-[3]. Singular Value Decomposition is utilized in [3] and applied to the Jacobian matrix to monitor trends toward singularity. A decoupled calculation of the Jaco- 
bian matrix yields highly accurate data while decreasing computational time dramatically by looking at the partial derivative of real power by bus angle and the partial derivative of reactive power with respect to voltage magnitude [4] [5]. In essence, analyzing the decoupled Jacobian matrix for singular values has been shown to be computationally faster and accurate, so a continuation of the decoupled Jacobian matrix is ideal. The theory adjacent to why singularities in the power flow Jacobian matrix yield unstable conditions clarifies why instability will occur. The decoupled Jacobian matrix approximates the change in real power at a bus due to bus angle and the change in reactive power at a bus due to bus voltage of all connected buses. If an eigenvalue of the Jacobian matrix, decoupled or full, approaches a singularity, meaning zero or infinity, that mathematically means that any change to voltage or bus angle will yield no change or an infinite change. In the case of a zero eigenvalue, that means that bus voltages or bus angles would need to be changed by an infinite amount before the complex power would fluctuate. In the case of an infinite element, any change to the bus angle or bus voltage would theoretically yield an infinite change to complex power. Only when the system is unstable should these conditions happen, so monitoring eigenvalues approaching singularity gives indication of system weak areas before instability occurs. In [4] [5], the decoupled Jacobian matrix is used over several Newton Raphson iterations to provide state estimations due to changes in bus angle and voltage. A Jacobian matrix containing singularity can no longer be used for power flow. Prony Analysis has been used to identify the slowest dominant system modes and the corresponding damping ratio from the eigenvalues correspondent to that mode [6]. Prony analysis also has been used alongside Frequency Domain Decomposition and oscillatory monitoring system approaches as in [6] [7]. This analytical method has been used to determine unstable conditions in slow tie-line power flows [6] and inter-area oscillations [8]. In [9] Prony analysis was applied to ring down data in real-time for a 17 machine model, showing its applicability for larger systems. However, for very large scale systems, real-time application becomes more difficult to implement. Utilizing Prony analysis on real power at each bus to monitor dominant system modes, eigenvalue poles, and associated damping ratios, gives valuable information in distinguishing a healthy system profile.

For a large scale system with $\mathrm{N}$-number of buses, extensive computational time is needed to perform power flow calculations. Since open PDC data lack system topology, model-free methods are necessary to utilize PMU data. Traditional power flow methods and state estimation require system connectivity and a long computational time to converge, rendering these approaches unsuitable for real-time open PDC data. This paper proposes a novel method, DDJEA that attempts to approximate the eigenvalues of the decoupled Jacobian matrix at each bus by measuring the difference between two neighboring measurements of real and reactive power with bus angle and voltage respectively. DDJEA's accuracy is analyzed to verify that it performs mathematically the same function as the full power flow Jacobian, while minimizing matrix size to incorporate only the number of PMUs. This reduction and simplification greatly decreases computation time. The DDJEA matrices are analyzed to ascertain parameters for stabil- 
ity and event detection. By looking at the elements of DDJEA as they approach a singularity, accelerating toward zero or infinity, system weak points can be flagged to operators before system instability occurs. On average for real open PDC samples, the speed of the proposed algorithm converges and outputs situational awareness data for 60 PMUs in 27 microseconds on a standard laptop. The DDJEA method is expanded in section 2.3 to ascertain divergent conditions and changes over time to monitor and flag both fast and slow unstable parameters. Prony analysis also is a powerful tool in identifying slow system dynamics that may cause instability if unresolved. Furthermore, dominant mode positive eigenvalues can be used to indicate fast unstable conditions. By comparing the event detection and instability indicators of both methods, strengths of the proposed method standalone are discussed, as well as a combined methodology to enhance situational awareness and stability monitoring.

\section{Decoupled Discreet-Time Eigenvalue Approximation Accuracy}

\subsection{Accuracy of Real Power State Prediction by Approximating $\Delta P_{i}$}

Over a short time period, assuming that the Jacobian matrix has not changed significantly between two cycles, the decoupled Jacobian matrix can be used to calculate changes in real power with respect to changes in bus angle. When a fault occurs or a significant load change, the error will increase, but this is addressed later for aiding event detection. The below formal definition of the decoupled Jacobian matrix for $\Delta P_{i}$ is presented below and derived to show how the DDJEA method simplifies calculations through direct computation. For any calculations, the total number of buses $(N)$ and the total number of PMUs $(n)$ are used.

$$
\Delta P_{i}=\sum_{j=1}^{N} \frac{\partial P_{i}}{\partial \delta_{j}} * \Delta \delta_{j} .
$$

$P_{i}$ is the actual real power at a bus at a particular measurement for a specific bus $(i)$. $\Delta P_{i}$ expresses a change between the most recent measurement and the prior measurement or iteration. $\Delta \delta_{j}$ represents the difference between the most recent bus angle measurement and prior bus angle measurement where $\delta_{j}$ is the bus angle at bus $(j)$.

$$
\frac{\partial P_{i}}{\partial \delta_{j}}=-\left|Y_{i j} V_{i} V_{j}\right| \sin \left(\theta_{i j}+\delta_{j}-\delta_{k}\right) \text {. }
$$

$Y_{i j}$ denotes the $Y_{\text {bus }}$ value for the element relating to the p.u. connectivity between the buses $(i)$ and $(j) . \theta_{i j}$ is the phase angle relative to $Y_{i j}$ in radians. $V_{i}$ and $V_{j}$ are the relative p.u. voltages of the buses $(i)$ and $(j)$ respectively.

When the time step between samples is decreased, the measured difference due to a bus angle in DDJEA will approach the value of the Jacobian element, as seen in Equation (3) and implemented in Equation (8). As the time step, $\Delta t$, is decreased, the effect on the power due to a particular bus angle more closely approximates the power flow Jacobian, similar to how numerical integration becomes more accurate with a smaller step size. For large time steps, this approximation method is highly inaccurate and inapplicable. However, with a $30 \mathrm{~Hz}$ rate of new state information from open PDC PMU data, this method becomes very accurate since the Jacobian matrix is unlikely to change 
almost instantaneously. Only a major change in load or event causes a significant shift in the values between cycles of the Jacobian. Since connectivity is not given in the open PDC format, the above equations cannot be applied per its formal definition. Therefore, the solution is simplified.

It is probable that if 60 PMUs are placed in a 3200 bus system that almost none of those PMUs are directly connected, rendering all non-diagonal terms zero without connectivity. Furthermore, state estimation would need to be applied in order to use the full Jacobian, a very time-consuming, heavily computational process that would waste the speed of the PMU data in large power systems. The proposed method directly calculates the change in power at a bus due to the change in that particular bus's angle for each diagonal element. Since other buses connected are not known, all other changes are approximated by incorporating all changes into the approximated eigenvalue as shown in Equation (8). By assuming this, significant errors in the DDJEA are expected when there is an event at a nearby connected bus that has no readings on it. However, soon after these errors decrease as the approximation accounts for the new parameters. The approximation of predicted real power for the next iteration is derived in Equation (4) with an expanded definition as presented in Equation (5). In Equation (4), the Jacobian of the previous system state is considered valid for one cycle. If time $(t+\Delta t)$ is considered the current sample, then the new change in bus angle is used in order to calculate what the system state should be if the Jacobian has not changed significantly. Power is calculated directly from the next system state. The following derivation shows the basis for DDJEA, keeping in mind that $(t+\Delta t)$ technically notates the present, or most recent, measurement.

$$
\begin{gathered}
\lim _{\Delta t \rightarrow 0} \frac{\Delta P_{i j}}{\Delta \delta_{j}}=\frac{\partial P_{i}}{\partial \delta_{j}} . \\
\Delta P_{i}(t+\Delta t)=\frac{\Delta P_{i}(t)}{\Delta \delta_{i}(t)} * \Delta \delta_{i}(t+\Delta t) . \\
\Delta P_{i}(t+\Delta t)=\frac{P_{i}(t)-P_{i}(t-\Delta t)}{\delta_{i}(t)-\delta_{i}(t-\Delta t)} *\left(\delta_{i}(t+\Delta t)-\delta_{i}(t)\right) .
\end{gathered}
$$

The term $\Delta P_{i}(t+\Delta t)$ represents the change in power at bus $(i)$ at the most current measurement. The term $P_{i}(t)$ is the real power of the last measurement. All calculations consider this notation.

$$
\begin{gathered}
\therefore P_{i}(t+\Delta t)_{\text {predicted }}=P_{i_{\text {acutal }}}(t)+\Delta P_{i}(t+\Delta t) . \\
P_{i}(t+\Delta t)_{\text {actual }}=3 * V_{1 i} * I_{1 i_{\text {total }}} * \cos \left(\delta_{V 1 i}-\delta_{I 1 i}\right) .
\end{gathered}
$$

The term $V_{1 i}$ represents the positive sequence voltage at bus (i). Likewise, $I_{1 i_{\text {total }}}$ represents sum of the positive sequence current through bus $(i)$.

$$
\left[\begin{array}{c}
\Delta P_{1}(t+\Delta t) \\
\vdots \\
\Delta P_{n}(t+\Delta t)
\end{array}\right]=\left[\begin{array}{ccc}
\frac{\Delta P_{1}(t)}{\Delta \delta_{1}(t)} & 0 & 0 \\
0 & \ddots & 0 \\
0 & 0 & \frac{\Delta P_{n}(t)}{\Delta \delta_{n}(t)}
\end{array}\right]\left[\begin{array}{c}
\Delta \delta_{1}(t+\Delta t) \\
\vdots \\
\Delta \delta_{n}(t+\Delta t)
\end{array}\right]
$$




$$
P_{\text {err }}=\frac{P_{\text {actual }}-P_{\text {predicted }}}{P_{\text {actual }}} * 100 .
$$

Essentially, this method generalizes to approximating the eigenvalues of the actual decoupled Jacobian matrix for each bus that has a PMU. Since it was previously assumed that most of the PMUs are not connected, all off diagonal terms are being calculated as zero. This leaves the DDJEA matrix in Equation (8) in eigenvalue form. Instead of needing to apply Singular Value Decomposition in order to determine singular values as done in [1]-[3], the DDJEA eigenvalues can be analyzed to assess if the matrix is approaching a singularity. In order to validate the proposed method, the percent error of DDJEA in Equation (9) with regard to predicting the next state is assessed in both simulation and in open PDC measurements. The IEEE 14 bus system is used for a 90 second simulation, 2700 samples at $30 \mathrm{~Hz}$. A three-phase fault is applied in the simulated system at bus 4 for 0.1 seconds, which clears by a line removal. This is used as a case study for which to assess the accuracy of the decoupled Discreet-Time Jacobian Eigenvalue Approximation for both real and reactive power. Measurements acquired from industry through open PDCare also assessed for accuracy of the method over a 147.5 second sample window, 4225 sample measurements. Equation (9) is used to derive the errors as shown in Table 1.

In Table 1, the mean perScent error for real power of the simulation is significantly higher than the median percent error. This is because during fault and during the line break, the DDJEA is highly inaccurate for a few measurements since particular DDJEA elements approach an infinite singularity during those immediate measurements, causing a significant error. These outliers can actually be used during event detection in the final algorithm to support that a major system event has occurred, as well as detect changes in system topology. The median percent error of simulation is practically non-existent. For the open PDC measurements of actual PMUs, the chaos in the system is significantly higher so the median percent error is higher. However, the mean percent error on predicting the next system state is still well under $0.5 \%$. Therefore, since the DDJEA method provides incredibly accurate measurements of the next system state utilizing the next iteration's change in bus angle, it can be seen that although the system connectivity is not known and all system buses are not used, DDJEA provides similar results by just approximating the eigenvalues of the decoupled Jacobian at each pmu and predicting the next state.

\subsection{Accuracy of Reactive Power State Estimation by Approximating $\Delta Q_{i}$}

The calculations for reactive power are very similar to the derivation for the decoupled

Table 1. DDJEA accuracy for real power estimation.

\begin{tabular}{ccc}
\hline \multirow{2}{*}{ Calculation Source } & \multicolumn{2}{c}{ Percent Error of Real Power $\left(P_{\text {err }}\right)$ for Measurements } \\
\cline { 2 - 3 } & Mean Percent Error $\left(P_{\text {err }}\right)$ & Median Percent Error $\left(P_{\text {err }}\right)$ \\
\hline IEEE 14 Bus Simulation & $0.054 \%$ & $9.51 \times 10^{-6} \%$ \\
Open PDC Measurements & $0.1977 \%$ & $0.1003 \%$ \\
\hline
\end{tabular}


method of estimating real power. In this case, $\Delta Q_{i}$ represents the change in reactive power from one measurement or iteration to the next. $Q_{i}$ is the reactive power at bus (i). Otherwise, all other notation in this section is the same as 2.1 .

$$
\begin{gathered}
\Delta Q_{i}=\sum_{j=1}^{N}\left|V_{j}\right| * \frac{\partial Q_{i}}{\partial\left|V_{j}\right|} * \mid \frac{\Delta V_{j}}{V_{j}} . \\
\frac{\left|V_{i}\right| \partial Q_{i}}{\partial\left|V_{j}\right|}=-\left|V_{i}\right|\left|Y_{i j} V_{i} V_{j}\right| \sin \left(\theta_{i j}+\delta_{j}-\delta_{k}\right) .
\end{gathered}
$$

The following derivations present how the PMU data can be used to approximate Equations (10) and (11), similar to the derivations presented for real power.

$$
\begin{gathered}
\Delta Q_{i}(t+\Delta t)=\frac{\Delta Q_{i}(t)}{\left|\Delta V_{i}(t)\right|} *\left|V_{i}(t)\right| * \frac{\left|\Delta V_{i}(t+\Delta t)\right|}{\left|V_{i}(t+\Delta t)\right|} . \\
\Delta Q_{i}(t+1)=\frac{Q_{i}(t)-Q(t-\Delta t)}{\left|V_{i}(t)-V_{i}(t-\Delta t)\right|} *\left|V_{i}(t)\right| * \frac{\left|\left(V_{i}(t+\Delta t)-V_{i}(t)\right)\right|}{\left|V_{i}(t+\Delta t)\right|} . \\
\therefore Q_{i}(t+\Delta t)_{\text {predicted }}=Q_{i}(t)+\Delta Q_{i}(t+\Delta t) . \\
Q_{i}(t+\Delta t)_{\text {actual }}=3 * V_{1 i} * I_{1 i_{\text {total }}} * \sin \left(\delta_{V 1 i}-\delta_{I 1 i}\right) . \\
\left.\left[\begin{array}{c}
\Delta Q_{1}(t+\Delta t) \\
\vdots \\
\Delta Q_{n}(t+\Delta t)
\end{array}\right]=\left[\begin{array}{ccc}
\frac{\Delta Q_{1}(t)}{\left|\Delta V_{1}(t)\right|} *\left|V_{1}(t)\right| & 0 & 0 \\
0 & \ddots & 0 \\
0 & 0 & \frac{\Delta Q_{n}(t)}{\left|\Delta V_{n}(t)\right|} *\left|V_{n}(t)\right|
\end{array}\right]|| \begin{array}{c}
\mid \frac{\Delta V_{1}(t+\Delta t)}{V_{1}(t+\Delta t) \mid} \\
\vdots \\
\left|\frac{\Delta V_{n}(t+\Delta t)}{V_{n}(t+\Delta t) \mid}\right|
\end{array}\right] . \\
Q_{\text {err }}=\frac{Q_{\text {actual }}-Q_{\text {predicted }} * 100 .}{Q_{\text {actual }}}
\end{gathered}
$$

DDJEA is used to approximate the next reactive power state for PMUs in the same IEEE 14 bus test system mentioned previously. Also the same 147.5 second open PDC measurements are used to ascertain DDJEA's $\Delta \mathrm{Q}_{\mathrm{i}}$ accuracy in a real-world application. Table 2 uses Equation (17) to derive the error of each reactive power state prediction.

In Table 2, the sample measurements yield that the mean percent error for predicting the reactive power state is less accurate than applying DDJEA to estimate the real power. Since the goal of the power grid is to supply consistent real power at all times, fluctuation of reactive power makes sense since the added variability would add some degree of error. However, the mean and median percent errors of DDJEA to both the

Table 2. DDJEA accuracy for reactive power estimation.

\begin{tabular}{ccc}
\hline \multirow{2}{*}{ Calculation Source } & Percent Error of Predicted and Actual Reactive Power State $\left(\mathrm{Q}_{\text {err }}\right)$ \\
\cline { 2 - 3 } & Mean Percent Error & Median Percent Error \\
\hline IEEE 14 Bus Simulation & $0.1884 \%$ & $9.104 \times 10^{-7} \%$ \\
Open PDC Measurements & $0.8916 \%$ & $0.3342 \%$ \\
\hline
\end{tabular}


simulated and real open PDC PMU measurements are well under 1\%. Therefore, the eigenvalue approximation for the change in reactive power due to the change in bus voltage is an effective estimate of the actual Jacobian's eigenvalue at each bus.

\subsection{Applying DDJEA for Situational Awareness and Stability Analysis}

In the traditional power flow Jacobian, Singular Value Decomposition is used in order to identify system weak points as they approach singularity [3]. In the proposed DDJEA method, SVD is not necessary because the matrix is already in eigenvalue form. It can be used to separate the matrix into zones of different magnitude, especially during an event, but it is more effective to analyze each bus individually and identify if the DDJEA matrix is approaching a singularity. If a singularity occurs, then the algorithm is set to flag the zones affected. However, it is more practical to flag the zone long before the singularity actually occurs so that the system operators know the area that is going unstable and have time to isolate or fix the issue. Knowing which buses are most negatively affected by a set of unstable parameters allows for more effective solutions by system operators, as well as knowing which buses or events are causing the problem. Instability due to non-convergent inter-area or tie line power flows can be solved by checking oscillations and changes in Jacobian eigenvalues, a problem which is also answered through Prony analysis. Monitoring the DDJEA gives an indication of how quickly the system parameters are changing and whether the system is converging or diverging due to power oscillations.

In order to determine whether the DDJEA is approaching a singularity, the characteristics of sequential DDJEA outputs are analyzed. A change in system topology or an event causes the DDJEA to change significantly, similar to how the power flow Jacobian changes frequently. If the DDJEA is increasing toward infinity or decreasing toward zero, it can be monitored. A consistent behavior that is sufficiently far from the mean value the either decoupled DDJEA matrix tends to take, accounting for standard deviation, causes system operators to be flagged. However, an additional tool has to be derived to ascertain when the DDJEA method is accelerating toward an instability point. After a significant system event such as a fault or line removal, system parameters that are quickly causing the system to go unstable need to be identified. Therefore, calculations to determine whether a bus's conditions are increasing/decreasing in Equation (19) and accelerating /decelerating in Equation (20) are developed. For terminology, this is called the acceleration indicator, AI.

$$
\begin{aligned}
A I(t)= & \Delta\left(\frac{\Delta P_{i}(t)}{\Delta \delta_{i}(t)}\right)=\left|\left(\frac{\Delta P_{i}(t)}{\Delta \delta_{i}(t)}-\frac{\Delta P_{i}(t-\Delta t)}{\Delta \delta_{i}(t-\Delta t)}\right)\right|-\mid\left(\frac{\Delta P_{i}(t-\Delta t)}{\Delta \delta_{i}(t-\Delta t)}-\frac{\Delta P_{i}(t-2 \Delta t)}{\Delta \delta_{i}(t-2 \Delta t)}\right) . \\
& |A I(t)|-|A I(t-\Delta t)|>0 \stackrel{\text { yields }}{\longrightarrow} \text { bus DDJA is accelerating. } \\
& \left(\left|\frac{\Delta P_{i}(t)}{\Delta \delta_{i}(t)}\right|-\left|\frac{\Delta P_{i}(t-\Delta t)}{\Delta \delta_{i}(t-\Delta t)}\right|\right)>0 \stackrel{\text { yields }}{\longrightarrow} \text { bus DDJA is increasing. }
\end{aligned}
$$

Similarly the same equations for reactive power can be derived. With these analytical 
tools, acceleration toward either an infinite or null singularity can be assessed and patterns can be determined long before a singularity is reached, resulting in voltage or angular instability depending upon which section of the decoupled Jacobian is approaching singularity. For situational awareness, the portion of the DDJEA approximating real power was most advantageous for showing events and fast system changes. The reactive power's relation to voltage is better for static analysis and assessing voltage stability as the voltage magnitude plays a large role in calculating those elements. Assessing the determinate of the full DDJEA as it approaches 0 is the key to determining a static approach to voltage stability [1]. The same principles apply to the decoupled variation of the power flow Jacobian [4] [5]. The flowchart in Figure 1 generalizes how the DDJEA method is used to relay situational awareness. For the simulation, all major events are recognized by a magnitude change of order 2 or more from the previous system measurement. When the DDJEA diverges from normal system operation and the absolute value begins to decrease to zero or increase toward infinity, indication of a slower instability condition arises and if the trend continues, system operators are flagged. If the DDJEA method begins to show an acceleration increase, then the location and parame-

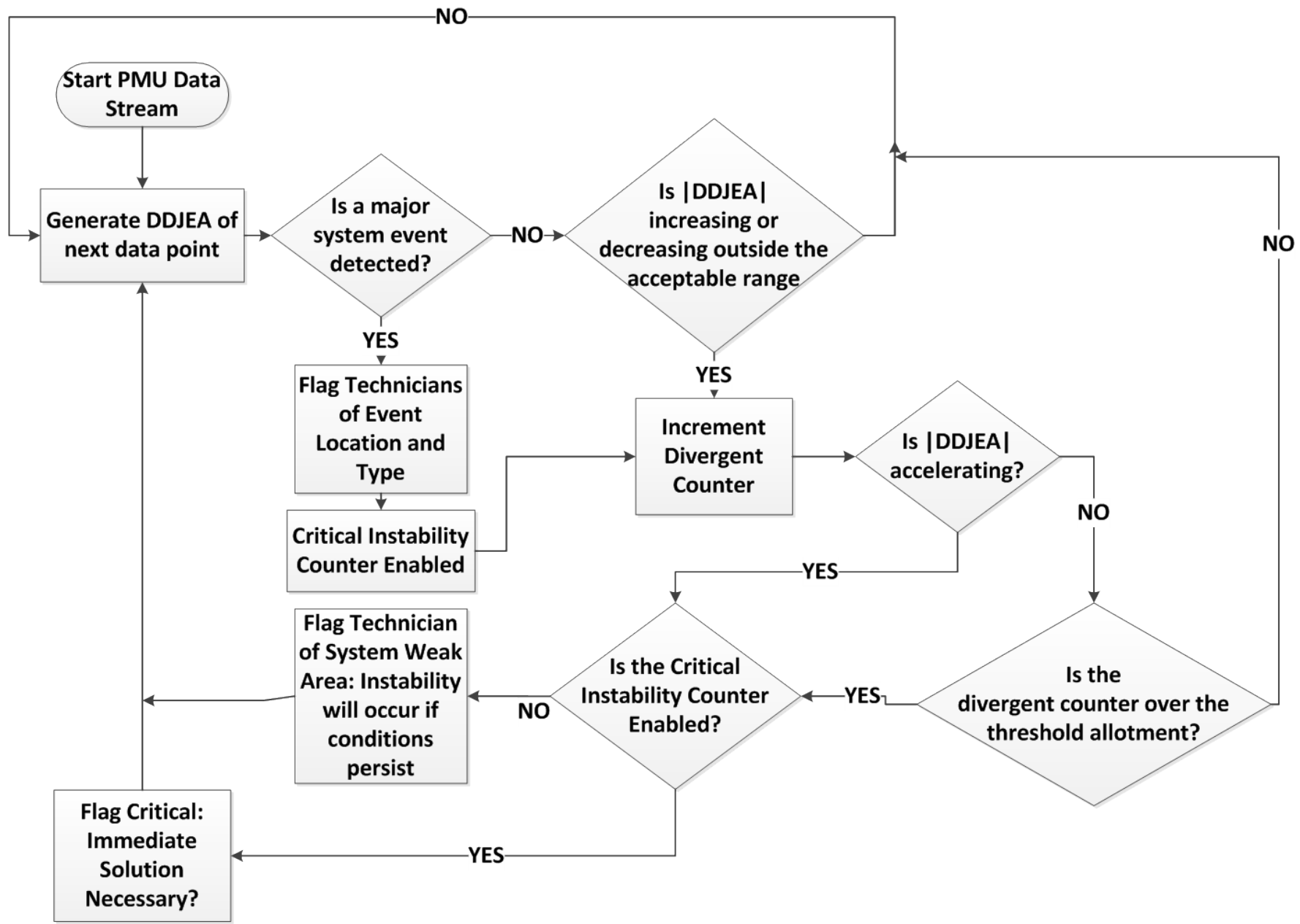

Figure 1. DDJEA interpretation method for situational awareness. 
ters are immediately sent to system operators since this means unstable conditions are approaching much faster than a slowly divergent system. By looking at the error of DDJEA, as well as how it changes, both event detection and a way to determine if an equilibrium point is being reached can be derived. The reactive portion of the decoupled Jacobian is a good way to check if an increase real power by bus angle is just the system reaching a new equilibrium point, resulting in no flags. Figure 1 also shows how the abundant data can be transformed through the DDJEA to give a fast indication of system weak points and unstable conditions. The next section will discuss how this approach, combined with Prony analysis, becomes an even more effective tool since both methods have their strengths.

When looking at the DDJEA application, it is important to note that situations which are accelerating outside of the normal system value range are immediately flagged. Looking at both eigenvalues associated with the decoupled matrices yield more information on event identification. However, this paper solely focuses on flagging unstable conditions and the most sensitive regions to changing parameters.

The algorithm in Figure 1 is implemented on an IEEE 14 bus system with unstable parameters and a fault in order to show the system weak point, which in this case is at the bus with a PMU notated as PMU 4. Different system classifications are assigned to each data point and described in Table 3 in order to interpret Figure 2 which presents the output to system operators. Loads were changed after the fault, which is not directly near PMU 4, such that if the system operates as intended, it will go unstable. It is important to note that the weak point of the system was not the nearest to the fault. Table 3 is used to decode the graph since each value means something different, and only specific values and thresholds should flag operators. In Figure 2, all prior measurements not shown had no flags, and a code value of 0 is given in Table 3 .

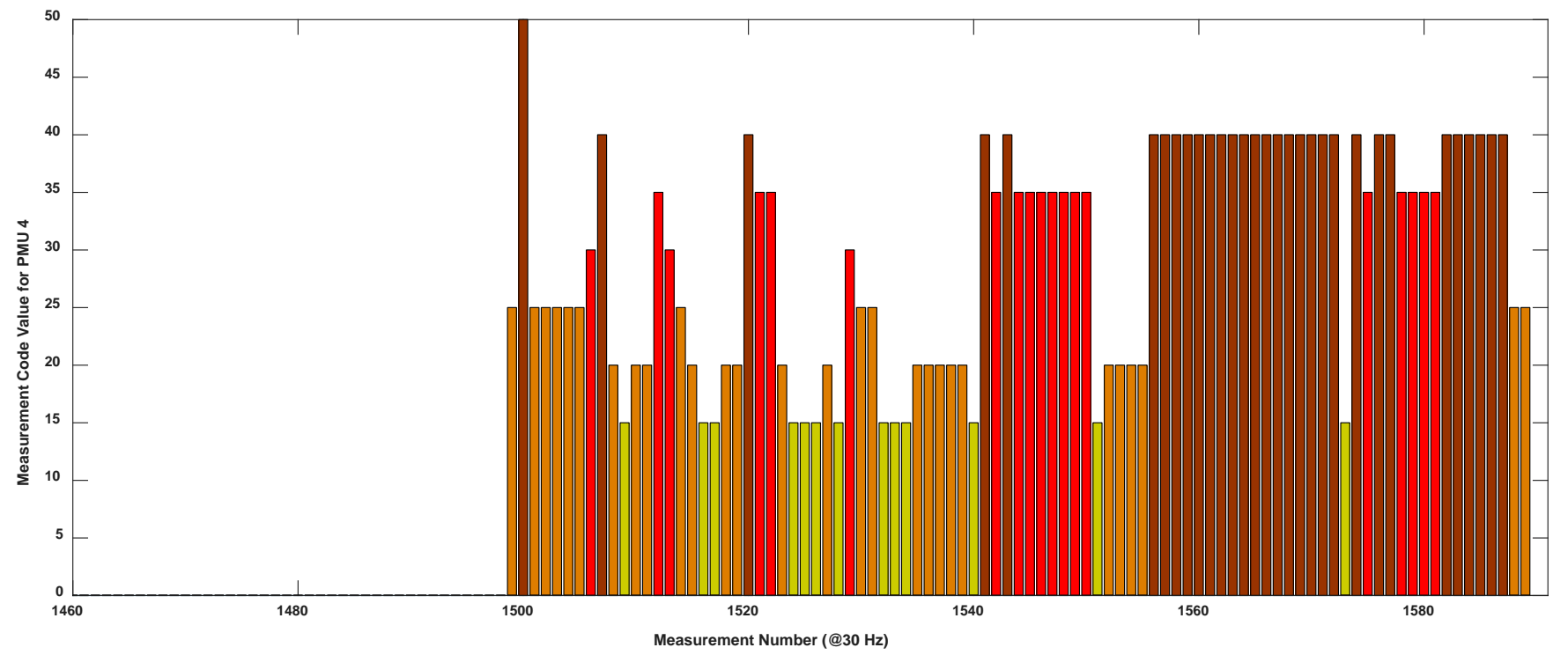

Figure 2. DDJEA analysis on simulated PMU data with unstable case. 
Table 3. DDJEA analysis code value meaning.

\begin{tabular}{cc}
\hline \multirow{2}{*}{$\begin{array}{c}\text { Code } \\
\text { Value }\end{array}$} & Figure 2 Decode Table \\
\cline { 2 - 2 } 5 & Interpretation of Value \\
\hline 10 & System is Fine/No action Necessary \\
15 & Slightly Divergent Trends Detected in Eigenvalue/No action unless this pattern continues \\
20 & Eigenvalue is Converging from Unstable Parameters/No action \\
25 & unless divergence occurs or after Event \\
30 & DDJEA Eigenvalue at Bus is Increasing toward Dynamic Instability/FLAG CRITICAL \\
35 & DDJEA Eigenvalue is Accelerating Toward Dynamic Instability/FLAG CRITICAL \\
40 & Approaching Singularity/ System will go unstable soon without solution/FLAG CRITICAL \\
50 & Major System Event/FLAG HIGHEST PRIORITY
\end{tabular}

When the fault is applied at the 1500th measurement, DDJEA detects it as a major event. All other system operations and load changes prior the event are not flagged, being sorted under normal system function. Essentially, any repeated value over 25 will eventually lead to instability if the system cannot converge, so any trend of 25 or over flags operators immediately. Changes in the DDJEA more indicative of dynamic instability and voltage collapse are flagged as a higher priority and the weak point(s) of the system are identified to help aiding situation system operators to avoid any abnormal situation.

\section{Prony Analysis and Case Study}

Prony analysis is another method which does not require system topology to yield viable data. Ultimately the goal is to derive a damping ratio and a dominant frequency from the signal of interest. It has been used before on open PDC measurements to identify poorly damped modes [7] and tie line oscillations [6]. In this case, the real power at each bus is the signal of interest. The end result of Prony analysis is shown below with $F(t)$ being the signal of interest. $M$ is the approximate number of system modes. The final derivation is shown below for visualization in Equations (21) (22).

$$
\begin{gathered}
F(t)=\sum_{j=1}^{M} \alpha_{j} * \mathrm{e}^{\sigma t} * \cos \left(2 \pi f_{j} t+\phi_{j}\right) . \\
F(t)=\sum_{i=1}^{M} \beta_{i} \mathrm{e}^{ \pm j \phi_{i}} \mathrm{e}^{\lambda_{i} t} .
\end{gathered}
$$

For the code, the signal is transformed into a $\mathrm{z}$-domain transfer function. Then that transfer function is converted over to state space. The eigenvalues of the A-matrix determine the frequencies of each mode and associated damping ratios. As mentioned before, one primary novelty of Prony analysis is the ability to determine undamped 
primary modes leading to such issues as inter-area and tie-line oscillation. On open PDC measurements, the method will determine the dominant mode, frequency aligned with that mode, and the damping ratio by looking at the real and imaginary components of the eigenvalue.

Figure 3 shows that for the real PMU data being received, the same dominant mode and similar damping ratios are repeatedly calculated for a healthy system. When Prony analysis is primarily used to calculate tie-line instability [6], the damping ratio is calculated from an eigenvalue that has a negative real part. The eigenvalue for this case is not in the unstable region. However for fast events, the eigenvalue will be reflected in the damping ratio as a negative number during the event where the pole is unstable and positive. In openPDC measurements received, no case where a tie-line oscillation causing instability has yet been observed. Prony analysis will be compared to the DDJEA on the same case in order to get some comparisons on faster characteristics.

In Figure 4, early output of the graph shows a similar trend to Figure 3. Dominant modes and frequencies should change slowly over time. At the point labeled Flag 1, the dominant mode and frequency change significantly. This is an indication of change in major system topology, but it may not be an indication of unstable conditions. Flag 2 alerts operators of an unstable pole, an indication of system instability and a solution needs to be found if the dominant frequency and damping ratio do not reach suitable values soon. At Flag 3, Prony analysis yields consistent positive eigenvalues, indicating fast system instability and immediate action being necessary. In Figure 5, implementing the Prony analysis output for situational awareness is explained for general use. Low damping ratios repeated over longer periods of time will flag for oscillatory power flows likely to cause instability. Also, positive eigenvalues are flagged as direct markers of instability for faster events. For slow events, a counter keeps track of damping ratios less than five percent. When the system's eigenvalues are consistently under five per-

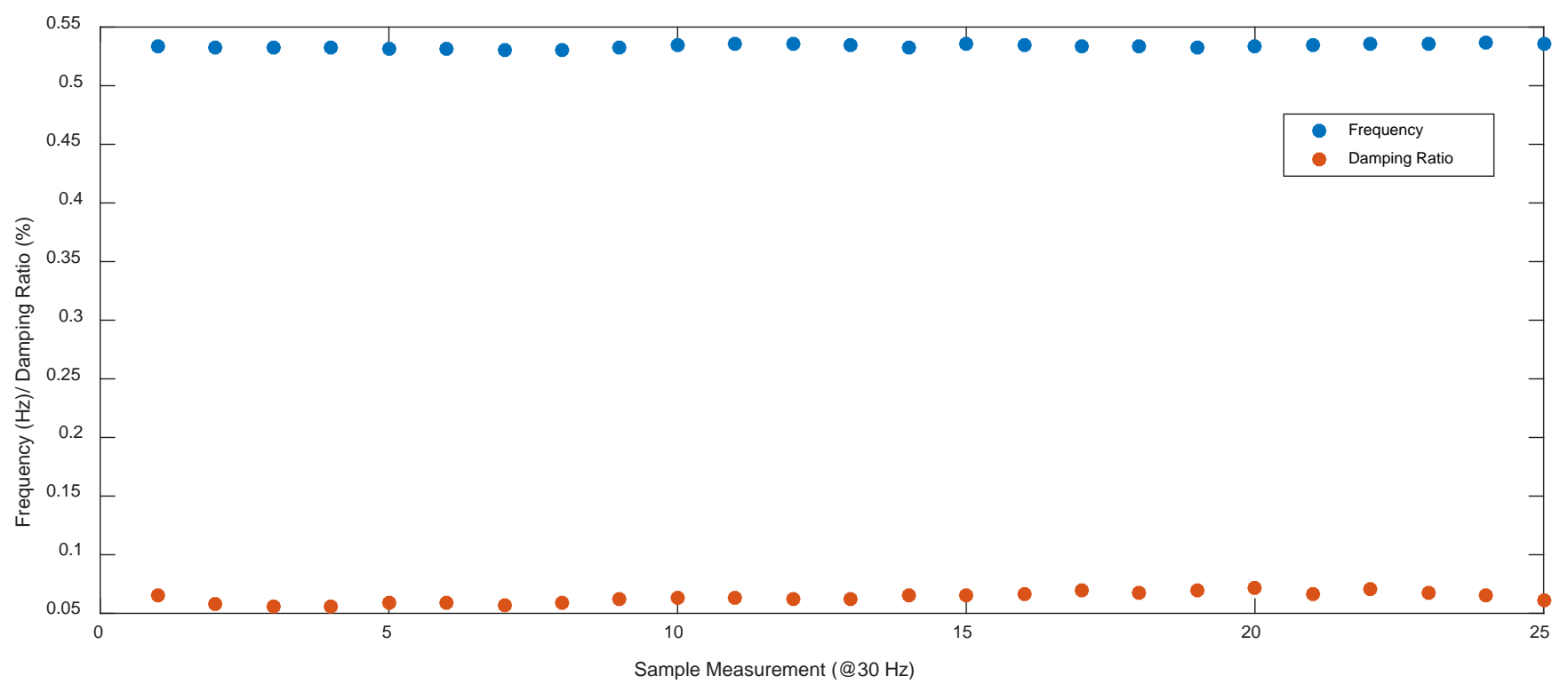

Figure 3. Prony analysis applied to open PDC. 


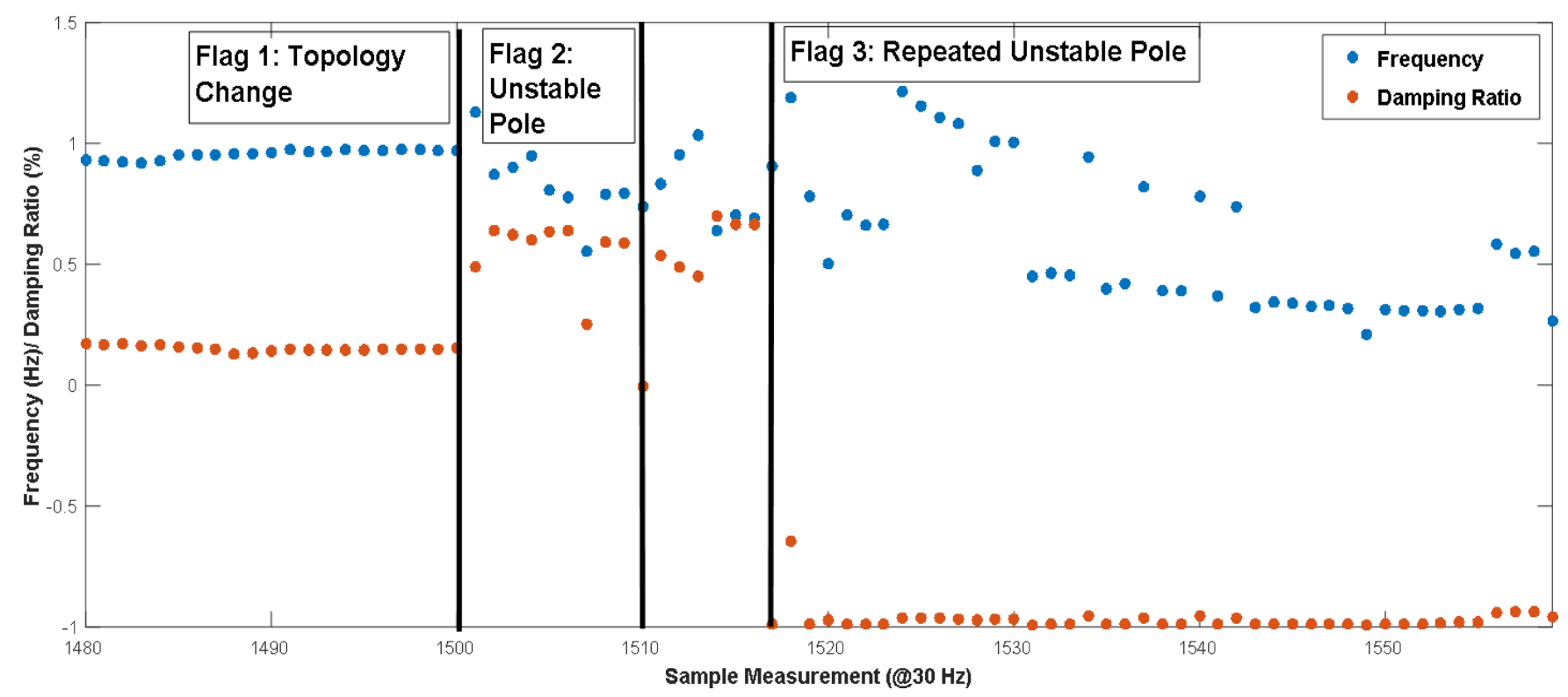

Figure 4. Prony analysis applied to unstable simulation.

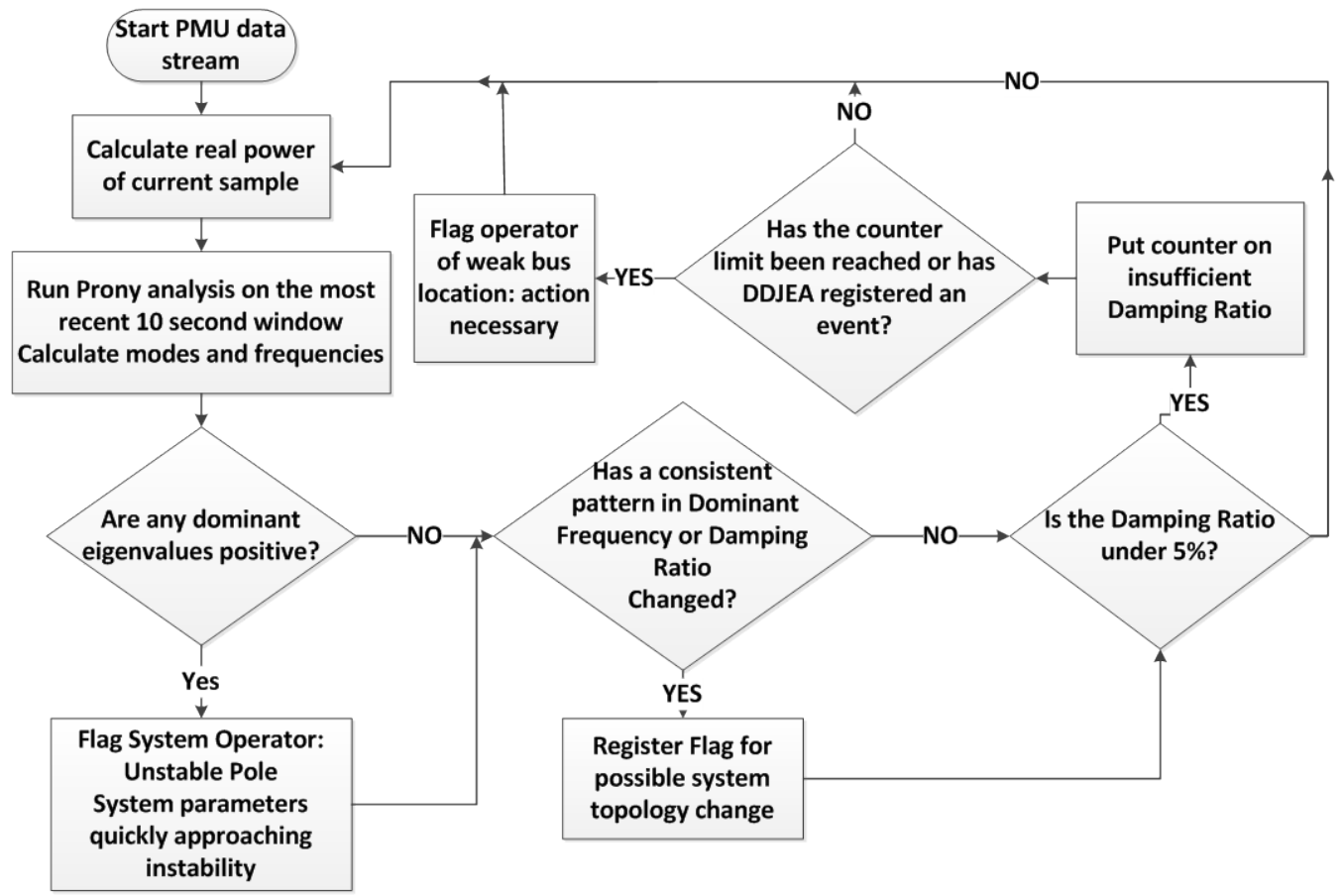

Figure 5. Prony analysis application for situational awareness (complimented with DDJEA).

cent, the system is not properly damped and some inter-area oscillation may be driving the system to instability.

\section{Comparison of Methods}

Although Prony analysis recognizes a change in system topology immediately, it doesn't fully recognize unstable conditions, while the DDJEA method does. Also, Prony 
analysis generates a high order pole transfer function from which results are derived. This is computationally intensive, whereas DDJEA can directly calculate values from PMU measurements. Data from 60 PMUs in openPDC for 147.5 seconds took the DDJEA method approximately 0.06 seconds to make all required calculations, easily qualifying it for real-time applications for very large systems. Depending on system dynamics, the computational time comparisons in Matlab placed the DDJEA method at around 6000 - 10,000 times faster than Prony analysis. Although Prony analysis requires a window of 10 seconds of data before it can run, in a real system it is an online application: a 10 second startup is negligible. DDJEA instantaneously reads in and calculates the next system state.

Depending on the data set, Prony analysis can be run to a full order model of every PMU; since DDJEA is so computationally light, running it adjacently would not hinder the process because it converges so quickly to a solution. In large data sets, DDJEA can be run continuously and Prony analysis can be run at a frequency that allows the overall solution to converge in real-time. In this way, damping ratios could be monitored to avoid missing an unstable power flow case which DDJEA can verify by checking if the eigenvalues have been approaching singularity, even if this change is very slow. Despite its computational expense, Prony analysis is a perfect tool for approximating system poles and modes. Due to the variability that a real power flow Jacobian can experience over time, also experienced by DDJEA methods, occasional Prony analysis iterations would be ideal as an adjacent tool to catch a slow poorly damped tie-line/inter-are oscillation. DDJEA is computationally faster algorithm that gives accurate estimates of the Jacobian eigenvalues without any system topology. Due to the speed at which the proposed algorithm can converge, there is plenty of room for more expansion of what the DDJEA algorithm can do and other methods that it could be coupled with. Table 4 makes some major comparisons between the two methods.

Table 4. DDJEA versus prony analysis.

\begin{tabular}{|c|c|c|}
\hline \multirow{2}{*}{ Topic of Interest } & \multicolumn{2}{|c|}{ Comparison of Methods } \\
\hline & DDJEA & Prony Analysis \\
\hline $\begin{array}{l}\text { Real-time } \\
\text { Applicability }\end{array}$ & $\begin{array}{l}\text { Yes, the method converges for } \\
\text { large systems before the next iteration. }\end{array}$ & $\begin{array}{l}\text { Yes and No } \\
\text { The method can be a real-time application } \\
\text { if the number of signals inputted } \\
\text { is reduced to converge in real-time. } \\
\text { It cannot converge for very large systems } \\
\text { unless the system order is reduced, } \\
\text { adding inaccuracy to the results. }\end{array}$ \\
\hline $\begin{array}{l}\text { Immediate Event } \\
\text { Detection }\end{array}$ & Yes & May take a few cycles \\
\hline $\begin{array}{c}\text { Slow Unstable } \\
\text { Power Oscillation } \\
\text { Detection }\end{array}$ & $\begin{array}{l}\text { It can detect eigenvalues increasing } \\
\text { toward singularity but may fail to flag as } \\
\text { immediately as Prony if matrices } \\
\text { appear to be approaching an } \\
\text { equilibrium point early on. }\end{array}$ & $\begin{array}{l}\text { Yes, this method excels } \\
\text { at this type of detection. }\end{array}$ \\
\hline
\end{tabular}




\section{Conclusion}

This paper presented an approximate method to be used for the purpose of situational awareness and assessing weak areas of the system without using system topology. The proposed DDJEA method used synchrophasor data to approximate the change in the system by observing the change in the diagonal terms of the DDJEA matrix, the approximation of the eigenvalues for the Jacobian matrix used in the Newton-Raphson power flow method. The accuracy of this novel approach was compared to what the power flow Jacobian matrix would yield, and the percent errors were all under one percent, showing the method as valid. In the case of an event, a comparison between the DDJEA method and the Newton-Raphson power flow calculations reached similar conclusions, giving indication of an unstable case and system weak areas in real time. The developed method was also compared with Prony analysis and results led to similar conclusions. However, the proposed method converged a lot faster and in a very short period after the event, which is a major factor when considering situational awareness. Future research will be extended to use of other techniques to enhance the proposed method by considering Gaussian distributions to give a better estimate of system connectivity and expand the DDJEA method closer to the full power flow Jacobian.

\section{Acknowledgements}

The authors would like to thank the members of Clemson University Electric Power Research Association (CUEPRA) for their financial support and providing PMU data.

\section{References}

[1] Sauer, P.W. and Pai, M.A. (1990) Power System Steady-State Stability and the Load-Flow Jacobian. IEEE Transactions on Power Systems, 5, 1374-1383.

http://ieeexplore.ieee.org/stamp/stamp.jsp?tp=\&arnumber $=99389$ http://dx.doi.org/10.1109/59.99389

[2] Bompard, E., Carpaneto, E., Chicco, G. and Napoli, R. (1996) A Dynamic Interpretation of the Load-Flow Jacobian Singularity for Voltage Stability Analysis. International Journal of Electrical Power \& Energy Systems, 18, 385-395.

http://ac.els-cdn.com/0142061595000828/1-s2.0-0142061595000828-main.pdf?_tid=88f8aee 4-55d5-11e6-9b87-00000aacb361\&acdnat $=1469828809 \_a 3 d 7830991205892$ bc2111fd63d57c $\underline{2 \mathrm{~d}}$ http://dx.doi.org/10.1016/0142-0615(95)00082-8

[3] Lof, P.-A., Smed, T., Andersson, G. and Hill, D.J. (1992) Fast Calculation of a Voltage Stability Index. IEEE Transactions on Power Systems, 7, 54-64.

http://ieeexplore.ieee.org/stamp/stamp.jsp?tp=\&arnumber $=141687$ http://dx.doi.org/10.1109/59.141687

[4] Stott, B. and Alsac, O. (1974) Fast Decoupled Load Flow. IEEE Transactions on Power Apparatus and Systems, 3, 859-869. http://dx.doi.org/10.1109/TPAS.1974.293985 http://ieeexplore.ieee.org/stamp/stamp.jsp?tp=\&arnumber=4075431

[5] van Amerongen, R.A.M. (1989) A General-Purpose Version of the Fast Decoupled Load Flow. IEEE Transactions on Power Systems, 4, 760-770.

http://ieeexplore.ieee.org/stamp/stamp.jsp?tp=\&arnumber $=193851$ 
http://dx.doi.org/10.1109/59.193851

[6] Guoping, L. and Venkatasubramanian, M. (2008) Oscillation Monitoring from Ambient PMU Measurements by Frequency Domain Decomposition. IEEE International Symposium on Circuits and Systems, Seattle, 18-21 May 2008, 2821-2824.

http://ieeexplore.ieee.org/xpl/articleDetails.jsp?arnumber $=4542044$ http://dx.doi.org/10.1109/iscas.2008.4542044

[7] Guoping, L., Ning, J., Tashman, Z., Venkatasubramanian, M. and Trachian, P. (2012) Oscillation Monitoring System Using Synchrophasors. IEEE Power and Energy Society General Meeting, San Diego, 22-26 July 2012, 1-8.

http://ieeexplore.ieee.org/stamp/stamp.jsp?tp=\&arnumber $=6345444$

[8] Bruelmann, H., Grebe, E. and Losing, M. (2000) Analysis and Damping of Inter-Area Oscillations in the UCTE/CENTRAL Power System.

http://citeseerx.ist.psu.edu/viewdoc/download;jsessionid=4C196EE579817D7A450EAA3A6 62DA104? doi=10.1.1.124.7846\&rep=rep1\&type $=$ pdf

[9] Ning, Z., Huang, Z., Tuffner, S., Pierre, J. and Jin, S. (2010) Automatic Implementation of Prony Analysis for Electromechanical Mode Identification from Phasor Measurements. IEEE PES General Meeting, Minneapolis, 25-29 July 2010, 1-8.

http://ieeexplore.ieee.org/xpls/abs_all.jsp?arnumber=5590169\&tag=1

http://dx.doi.org/10.1109/PES.2010.5590169

Submit or recommend next manuscript to SCIRP and we will provide best service for you:

Accepting pre-submission inquiries through Email, Facebook, LinkedIn, Twitter, etc.

A wide selection of journals (inclusive of 9 subjects, more than 200 journals)

Providing 24-hour high-quality service

User-friendly online submission system

Fair and swift peer-review system

Efficient typesetting and proofreading procedure

Display of the result of downloads and visits, as well as the number of cited articles

Maximum dissemination of your research work

Submit your manuscript at: http://papersubmission.scirp.org/ 